\title{
Practical Attacks on a Cryptosystem Proposed in Patent WO/2009/066313 ${ }^{\star}$
}

\author{
Gautham Sekar ${ }^{\star \star}$ and Bart Preneel \\ 1 Department of Electrical Engineering ESAT/SCD-COSIC, \\ Katholieke Universiteit Leuven, Kasteelpark Arenberg 10, B-3001 Heverlee, Belgium. \\ 2 Interdisciplinary Institute for BroadBand Technology (IBBT), Belgium. \\ \{Gautham.Sekar,Bart.Preneel\}@esat.kuleuven.be
}

\begin{abstract}
A new cryptosystem is proposed in the international patent WO/2009/066313 by Artus. The symmetric-key primitive in the cryptosystem resembles a stream cipher. The system differs markedly from cryptosystems used today in that the secret key is changed with the plaintext. The patent does not discuss key management (generation, transmission) in sufficient detail. Some of the proposed methods for transmission of keys are highly insecure and an algorithm for key generation is missing. In this paper, we find that related-key attacks of negligibly low complexity (data/time) can result when certain (flawed) key generation algorithms are used. We also present a negligibly-low-complexity attack in a non-related-key setting. We hope that the results caution potential users of the cryptosystem.
\end{abstract}

Keywords: Cryptosystem, stream cipher, public-key cryptography, chaffingand-winnowing, key management, cryptanalysis, related-key attack

\section{Introduction}

A new cryptosystem is proposed by Artus in [2]. The design is covered by the following patents: international (WO/2009/066313), US (US 2010/0153723), European (EP 2183875), Australian (AU 2008327506), Canadian (CA 2695019), Indian (1456/MUM/2007) and Russian (national reference number 2010104728). The Russian patent document is not yet publicly available. So far, to the best of our knowledge, there is no cryptanalysis result on this cryptosystem in the open literature. As the text (especially, cryptology parlance) is not entirely clear in [2], we base this paper on our best possible interpretation of it.

The cryptosystem (as it is not named in [2], we call it S) consists of a key distribution mechanism and a symmetric-key primitive. The latter is a stream cipher-like construction; for conciseness, henceforth, we shall call it a stream cipher. The system significantly differs from cryptosystems used today. The secret

\footnotetext{
* This work was supported in part by the IAP Program P6/26 BCRYPT of the Belgian State (Belgian Science Policy), and in part by the European Commission through the ICT program under contract ICT-2007-216676 ECRYPT II.

** This author is supported by an adapID project.
} 
key of the stream cipher is changed with every new message (plaintext). It is not clear to us what the designer means by 'new message' - there is no specific message length mentioned in [2]. The designer proposes some means for the initial key exchange between the sender and the receiver (e.g., via SMS, without any mention about encryption) and assumes that these are secure. Even though the designer notes that one could use "other means" (see [2]) to securely initiate communication, there is not a single mention of public-key cryptography (PKC).

Besides, the patent just mentions that the keys are changed in a random manner but fails to mention how. Performance estimates are also missing. Since keys are changed so often, the key generation algorithm and performance figures are extremely vital. Also required in [2] are the targeted applications for which the cryptosystem has been designed, and a more detailed security evaluation. Looking at the design, we presume that the proposed system is intended for software-oriented applications.

In this paper, we first point out problems associated with some of the proposed methods in [2] to initiate communication between the sender and the receiver. Following this, we present other attacks assuming that PKC is used to exchange the initial keys. The attacks work in both a related-key setting and otherwise, under some reasonable assumptions. Most of these assumptions have been made due to the absence of sufficient details in [2]. This and the fact that our assumptions are reasonable would be understood from the following sections.

In the non-related-key setting, we present an attack that recovers one plaintext bit from the corresponding ciphertext bit in nearly zero time. This attack results from the way in which decimal-to-binary conversions are performed in [2]. When decimal numbers are converted to binary and stored in (fixed-length) registers, some of the most significant bits may be zeroes (we call them leading zeroes). In [2], leading zeroes are discarded while converting decimal numbers to binary, thereby ensuring that the MSBs of the (truncated) binary numbers are all ones.

The related-key attacks result when certain key generation algorithms are used (the patent does not provide any algorithm for key generation). Each of these attacks requires only 2 plaintext-ciphertext pairs ${ }^{3}$ and negligible time to recover a key or key-dependent information with guaranteed success. As the key is changed with every new message, related-key attacks are important to be addressed. In a way, this paper tells one how not to use the proposed cryptosystem, especially with respect to key management.

Another motive behind this work is to caution potential users of the cryptosystem. We believe that they would interpret the unclear description of the cryptosystem in the same way as we have done in this paper. The practical nature of the attacks presented in this paper seems to suggest that WO/2009/066313 has no industrial applicability, as opposed to what the international search report of the patent claims. Given that the system is covered by 7 patents, it may not be unreasonable to imagine that there would be several potential buyers.

${ }^{3}$ Here, the term plaintext or ciphertext may have a connotation. This point is explained further in the paper. 
To further substantiate this point, we take the example of KeeLoq [11]. The block cipher KeeLoq is a proprietary algorithm used in remote keyless entry systems. It was designed in South Africa in the mid 1980's by researchers who had little or no prior record of designing cryptographic algorithms. The cipher was sold to Microchip Technology Inc. and subsequently used by companies such as Chrysler, Daewoo, Fiat, General Motors, Honda, Toyota, Volvo, Volkswagen Group, Clifford and Jaguar [11,17]. In the last few years, several researchers had analysed the cipher [17], and a practical attack was published in 2008 [7].

Organisation: The paper is organised as follows. Section 2 lists the notation and convention used throughout the paper. In Sect. 3, we detail the specifications of the cryptosystem S. In Sect. 4, we discuss the issue of initial key exchange between the sender and the receiver. Assuming the exchange has been performed securely, Sect. 5 presents some observations which are converted into practical attacks in Sect. 6. Finally, we conclude in Sect. 7 where we also provide some directions for future work.

\section{Notation and Convention}

The notation and convention used in this paper are listed in Table 1.

Table 1. Notation and convention

\begin{tabular}{|c|l|}
\hline Symbol / Notation & Meaning \\
\hline$\oplus$ & Bitwise XOR \\
\hline$\|$ & Concatenation \\
\hline LSB & Least significant bit \\
\hline MSB & Most significant bit \\
\hline$\beta_{i(x)}$ & Bit $x$ of $\beta_{i}, x=0$ denotes the LSB of $\beta_{i}$ \\
\hline$\beta_{i(x)}^{c}$ & Bit-complement of $\beta_{i(x)}$ \\
\hline$l e n(m)$ & Length of $m$ in bits ${ }^{\dagger}$ \\
\hline KP & Known plaintext \\
\hline CP & Chosen plaintext \\
\hline
\end{tabular}

${ }^{\dagger}$ It is not mentioned in [2] whether the leading zeroes are discarded while coding $l e n(m)$ in binary

\section{Description of the Cryptosystem}

The stream cipher (as it is not named in [2], we call it E) uses keys that are sufficiently long to thwart brute force attacks. The designer, however, does not recommended any particular key size. The internal state of E consists of an array 
$A$ that stores $N$ digits chosen arbitrarily from the decimal representation of $\pi$. We denote the array by $A[0, \ldots, N-1]$. It is not mentioned in [2] whether this array is secret or not. Despite this, we present in this paper attacks that recover the secret key or plaintext without the attacker knowing $A[0, \ldots, N-1]$. The key (or seed) is a 4 -tuple $\left(K_{1}, K_{2}, K_{3}, K_{4}\right)$ where:

1. $K_{1}$ is an arbitrarily chosen array index such that $0 \leq K_{1} \leq N-1$,

2. $K_{2}$ is a number that uniquely corresponds to a subset of $r$ elements $(r \geq \sqrt{N})$ of $A[0, \ldots, N-1]$ (i.e., $1 \leq K_{2} \leq C(N, r)$ ),

3. $K_{3}$ is a number that uniquely corresponds to an ordering of the $r$ elements determined by $K_{2}$ (i.e., $1 \leq K_{3} \leq r$ !)

4. $K_{4}$ determines how consecutive decimal digits in the $r$-element ordered list are concatenated; $1 \leq K_{4} \leq 2^{r-1}$.

We call each of these four components of the seed as subkey. According to [2], the seed is "dynamically" changed, in a random manner, for every message sent. To initiate communication between the sender (Alice) and the receiver (Bob), a mechanism is proposed. The text in the paper, that describes this mechanism, is not entirely clear to us and we believe that the designer may have been inspired by chaffing-and-winnowing [14]. The technique proposed in [2] does not employ a MAC algorithm; so it is definitely not chaffing-and-winnowing.

Alice first sends a file (a pilot-file according to [2]) to Bob. This file contains a long string of random-looking numbers of which the initial seed is a part. The following assumption is made by the designer.

Assumption 1: An algorithm to locate the initial seed, using four numbers, in the string of random numbers of the pilot-file is known to both Alice and Bob, but not to an eavesdropper (Mallory).

We denote the Algorithm in Assumption 1 by $L$ and the four numbers by the tuple $\left(\alpha_{1}, \alpha_{2}, \alpha_{3}, \alpha_{4}\right)$. This 4 -tuple is now sent by Alice, separately, by "Text, SMS or other means" [2].

The seed for the second message is encrypted using the first seed and then sent to Bob. The seed for the third message is encrypted using the second seed, and so on.

Figure 1 shows the working of the cipher E. The key $\left(K_{1}, K_{2}, K_{3}, K_{4}\right)$ first produces an ordered decimal number list (ODNL). The numbers in the list are converted to binary and the resultant bit strings are concatenated to give a single concatenated bit string (CBS). Let extended message (plaintext) denote the message (plaintext) concatenated with the seed that is intended to be used to encrypt the next message. The CBS is truncated if it is longer than the extended message. ${ }^{4}$ The (truncated) keystream is then XOR-ed with the extended message to yield the extended ciphertext. Figure 2 illustrates, with an example, the generation of the extended ciphertext. The extended ciphertext is split into two parts of variable lengths. A concatenation is then performed, where the length

\footnotetext{
${ }^{4}$ Details on how the truncation is done are missing in [2].
} 
(in bits) of the plaintext is placed between the two parts of the extended ciphertext. The resultant bitstream is transmitted to Bob. It is assumed by the designer that Bob (not Mallory) knows

- where the length of the plaintext is placed, i.e., the starting point of the bit string representing the length of the plaintext (starting point, hereafter) in the bit string of the extended ciphertext,

- the length (in bits) of the length of the plaintext.

Again, it is not mentioned in [2] whether the leading zeroes are discarded while coding the length of the length of the plaintext (from the length of the plaintext) in binary. Since it is an algorithm that is a part of S, by the Shannon's maxim [16], it is known to Mallory. Similarly, Mallory knows whether the leading zeroes are discarded while coding the length of the plaintext (from the plaintext) in binary.

Figure 3 shows the working of the cryptosystem $\mathrm{S}$ with a fixed starting point that immediately follows the LSB of the extended ciphertext. In this figure, $E_{\beta_{1}}\left(m_{1} \| \beta_{2}\right)$ is the output of stream cipher encryption, using the key $\beta_{1}$, of the message $m_{1}$ concatenated with the key $\beta_{2}$ (which is used to encrypt the next message $m_{2}$ ).

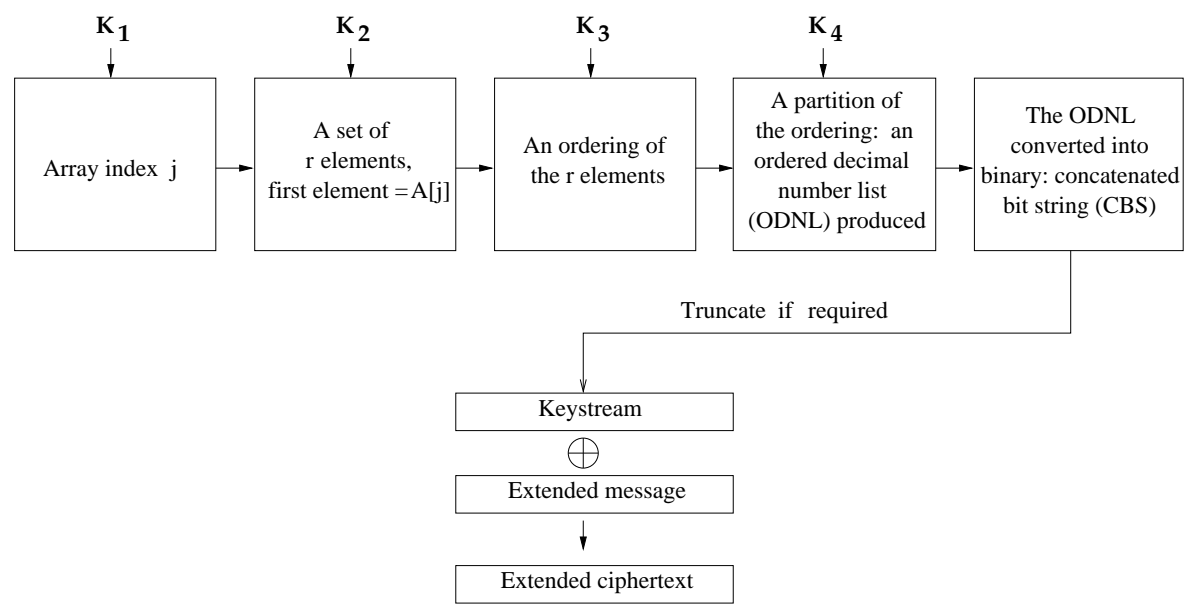

Fig. 1. Encrypting a plaintext message with E under key $\left(K_{1}, K_{2}, K_{3}, K_{4}\right)$

\section{A Trivial Attack on the Cryptosystem S}

In this section, we outline a trivial attack on the cryptosystem S. Recall that the algorithm $L$ is known only to Alice and Bob. A risk that this poses is when the secret algorithm is reverse engineered later and found to be insecure. For 


\section{$\pi=3.141592653589793238462643383279502884197169399375 \ldots$}

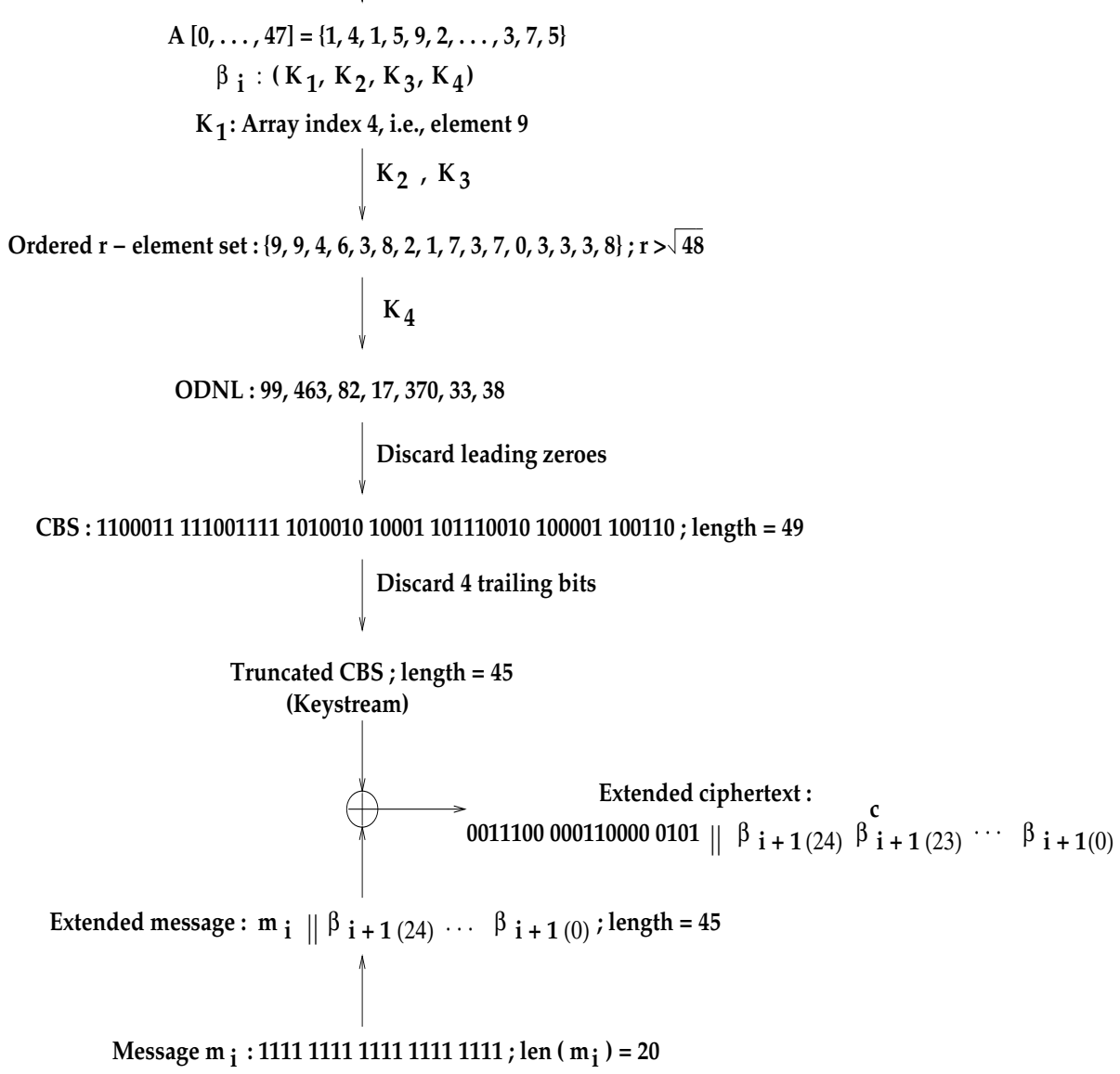

Fig. 2. An example (similar to the example in [2]) to illustrate the generation of the extended ciphertext; in this example, while truncating the CBS, the redundant trailing bits are discarded (while 4 leading bits could have instead been discarded) 


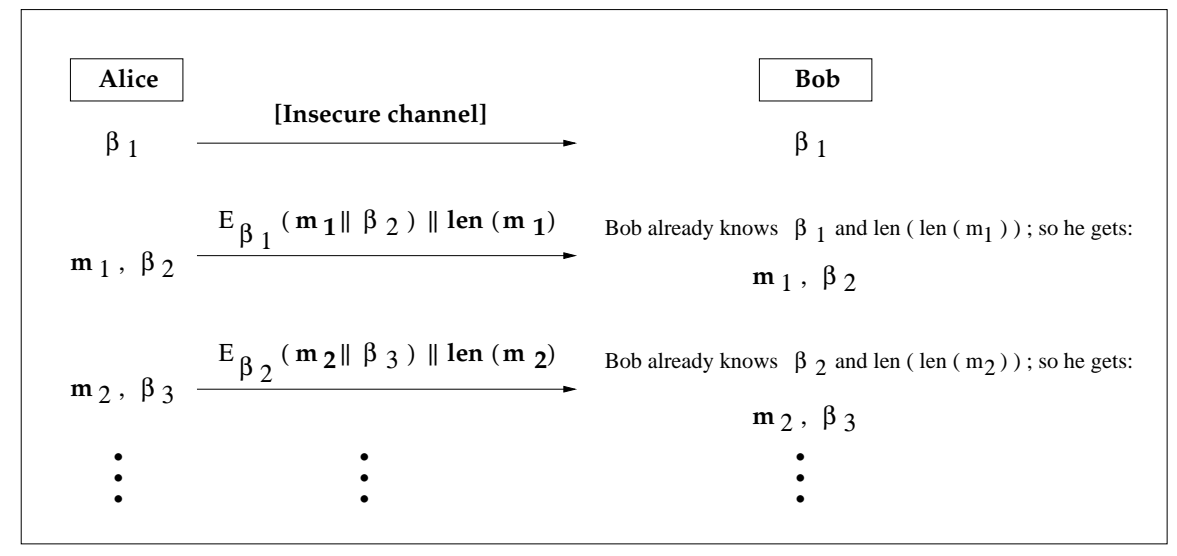

Fig. 3. Working of the cryptosystem $\mathrm{S} ; \beta_{1}$ may be securely transmitted across the insecure channel through the use of PKC

example, the stream cipher RC4 was a trade secret for several years before it was reverse engineered in 1994 and leaked to the Cypherpunks mailing list and the Usenet newsgroup sci.crypt [1]. Following this, the cipher witnessed several attacks some of which are listed in [10].

By the Kerckhoffs' principle [8], $L$ should not be required to be secret. If $L$ is known to Mallory, then she can obtain the initial seed from the pilot-file using $\left(\alpha_{1}, \alpha_{2}, \alpha_{3}, \alpha_{4}\right)$. The pilot-file and $\left(\alpha_{1}, \alpha_{2}, \alpha_{3}, \alpha_{4}\right)$ may be obtained by Mallory if these are sent through an insecure channel by Alice without encrypting properly. This sometimes applies to, for example, sending raw data by SMS. Though GSM phones support ciphers like A5/1, A5/2 and A5/3, in countries like France, India and Libya, encryption is disabled. Besides, practical attacks have been found in the A5/1 and A5/2 ciphers $[3,6]$. Recently, Dunkelman et al. claim to have found a practical-time attack on the A5/3 cipher [5]. Messaging via SMS has some other security vulnerabilities due to its store-and-forward feature.

The problem can be overcome if PKC (Diffie-Hellman protocol [4], RSA [13], or elliptic curve cryptography $[9,12]$ ) is one of the "other means" (see Sect. 3) of communicating the initial seed to Bob in [2]. Chaffing-and-winnowing is another technique that could be used to achieve confidentiality without performing encryption. However, if this technique were to be used, a MAC algorithm is required (HMAC-SHA1 has been suggested in [14]) and its secret key is shared by the sender and receiver using PKC (e.g., authenticated Diffie-Hellman key exchange). Chaffing-and-winnowing is primarily used to thwart attempts by law enforcement to regulate confidentiality by regulating encryption.

Even when the initial keys are exchanged securely, regardless of the technique used, we find the system $\mathrm{S}$ to contain serious weaknesses. We describe these in Sects. 5 and 6. 
In this paper, we construct a couple of attacks (in Sect. 6) under the following assumption.

Assumption 2: The bit string representing the length of the plaintext trails the LSB of the extended ciphertext.

With every message, if Alice were to provide the corresponding starting point securely to Bob, then she needs to use PKC. But doing so would make the scheme very slow for practical usage. To solve this, Alice and Bob can, alternatively, agree on one fixed starting point - for example, immediately following the LSB of the extended ciphertext (as in Figure 3). To agree on a single starting point value, Alice can enter it into the pilot file, extend the tuple $\left(\alpha_{1}, \alpha_{2}, \alpha_{3}, \alpha_{4}\right)$ to $\left(\alpha_{1}, \alpha_{2}, \alpha_{3}, \alpha_{4}, \alpha_{5}\right)$ and modify the algorithm $L$ accordingly. Now,

- if the pilot file and the 5 -tuple $\left(\alpha_{1}, \alpha_{2}, \alpha_{3}, \alpha_{4}, \alpha_{5}\right)$ are sent to Bob through an insecure channel without encrypting properly (e.g., via SMS), then Mallory may obtain the starting point when she knows $L$.

- Assume that PKC is used to transmit the pilot file and $\left(\alpha_{1}, \alpha_{2}, \alpha_{3}, \alpha_{4}, \alpha_{5}\right)$. Even then, for a transmitted bit string of length $d$, the probability that Mallory correctly guesses the starting point, without knowing the length of the plaintext, is at least $1 / d$. The probability is high when $d$ is small.

- In the preceding case, if Mallory knows the length of the plaintext $(\operatorname{len}(\mathrm{m})$, say), then she can search for the bit string in the string of bits transmitted by Alice. There are at most $d-\operatorname{len}(\operatorname{len}(m))+1$ matches $;{ }^{5}$ therefore, the probability that Eve correctly guesses the starting point is at least $1 /(d-$ len $(\operatorname{len}(m))+1)$. Again, the probability is high when $d$ is small or when the message $m$ is long.

In these scenarios, therefore, Assumption 2 is reasonable. But even if this assumption is not made, it is possible to attack S. We present this attack in Sect. 6 .

\section{Motivational Observations}

We continue our analysis, starting with the following observations. Let $m_{i}$ and $\beta_{i}(i \geq 1)$ respectively denote the $i$-th message and the seed used to encrypt it. The initial seed is $\beta_{1}$.

Observation 1: Let $\beta_{i} \leftarrow\left(K_{1}, K_{2}, K_{3}, K_{4}\right)$ and $\beta_{j} \leftarrow\left(K_{1}, K_{2}, K_{3}, K_{4}^{\prime}\right)$, where $K_{4}^{\prime} \neq K_{4}$. Then, regardless of relation between $K_{4}$ and $K_{4}^{\prime}$, the LSBs of the CBSs produced by the two keys are identical.

Observation 2: Let us consider the following two scenarios.

\footnotetext{
5 This upper bound is reached only when the transmitted bits are all ones or all zeroes; hence, one expects fewer matches. Our analysis, therefore, is worst-case with respect to the attacker.
} 
Scenario 1: Consider $\beta_{i} \leftarrow\left(K_{1}, K_{2}, K_{3}, K_{4}\right)$ and $\beta_{j} \leftarrow\left(K_{1}, K_{2}^{\prime}, K_{3}^{\prime}, K_{4}^{\prime}\right), j>i$, where $K_{2}^{\prime}, K_{3}^{\prime}$ and $K_{4}^{\prime}$ are such that the ODNL obtained using $\beta_{j}$ is a list that is formed by appending numbers to the ODNL obtained using $\beta_{i}$. Let $\operatorname{len}\left(m_{i} \| \beta_{i+1}\right)=\operatorname{len}\left(m_{j} \| \beta_{j+1}\right)$. Then, we have the following observation.

Observation: If the truncation of the CBS is such that the redundant trailing bits are discarded, the keystreams corresponding to $\beta_{i}$ and $\beta_{j}$ are identical.

Scenario 2: Consider $\beta_{i} \leftarrow\left(K_{1}, K_{2}, K_{3}, K_{4}\right)$ and $\beta_{j} \leftarrow\left(K_{1}^{\prime}, K_{2}^{\prime}, K_{3}^{\prime}, K_{4}^{\prime}\right), j>i$, where $K_{1}^{\prime}, K_{2}^{\prime}, K_{3}^{\prime}$ and $K_{4}^{\prime}$ are such that the ODNL obtained using $\beta_{j}$ is a list that is formed by prefixing numbers to the ODNL obtained using $\beta_{i}$. Let $\operatorname{len}\left(m_{i} \| \beta_{i+1}\right)=\operatorname{len}\left(m_{j} \| \beta_{j+1}\right)$. Then, we have the following observation.

Observation: If the truncation of the CBS is such that the redundant leading bits are discarded, the keystreams corresponding to $\beta_{i}$ and $\beta_{j}$ are identical. ${ }^{6}$

Observation 3: Given Assumption 2, if $\beta_{i}$ and $\operatorname{len}\left(\operatorname{len}\left(m_{i}\right)\right)$ are known to Mallory, she can obtain $m_{i}$ and $\beta_{i+1}$ (see Figure 3).

\section{Attacks Due to Weaknesses in the Stream Cipher}

In this section, we present several attacks on the cryptosystem that stem from flawed key management techniques and weaknesses in the underlying stream cipher. We use the observations of Sect. 5 .

\subsection{Attacks in a Related-Key Setting}

In a related-key setting, we present the following two attacks.

Attack 1: In this attack, Mallory obtains key-dependent information from message-dependent information. We begin with Observation 1. Suppose that the identical LSBs (denote them by $b$ ) of the CBSs produced by $\beta_{i}$ and $\beta_{j}$ are in the keystream (i.e., only the redundant leading bits of the CBS are discarded). ${ }^{7}$ Given Assumption 2, if $\operatorname{len}\left(\operatorname{len}\left(m_{i}\right)\right)$ and $\operatorname{len}\left(\operatorname{len}\left(m_{j}\right)\right)$ are known to Mallory, she knows $\beta_{i+1(0)} \oplus b$ and $\beta_{j+1(0)} \oplus b$. From these two values, she obtains key-dependent information in the form of $\beta_{i+1(0)} \oplus \beta_{j+1(0)}$. Often, in practice, Mallory knows len $\left(m_{i}\right)$ or $m_{i}$, so she can compute len $\left(\operatorname{len}\left(m_{i}\right)\right)$. Therefore, to mount this attack, Mallory only requires the lengths of two plaintexts, or 2 KPs and the corresponding intercepted texts (i.e., extended ciphertexts concatenated

\footnotetext{
${ }^{6}$ Since [2] does not discuss how the truncation of the CBS is done, it is not possible to tell which of the two scenarios results in identical keystreams. This is also the reason why we rule out the possibility that bits are discarded from the middle of a CBS.

7 The manner in which the CBS is truncated would normally be a part of the algorithm that is known to the attacker; hence, it is reasonable to assume that Mallory knows whether the leading or the trailing bits are discarded.
} 
respectively with the lengths of the plaintexts).

Attack 2: This attack works as follows. Consider the case when $\beta_{1}$ and $\beta_{2}$ are such that the keystreams they produce are identical (i.e., as in Observation 2). First, Mallory collects $E_{\beta_{1}}\left(m_{1} \| \beta_{2}\right)$, using the knowledge of len $\left(m_{1}\right)$ or $m_{1}$ itself. Now, Mallory forces Alice to encrypt a message of Mallory's choice, of length equal to $\operatorname{len}\left(m_{1} \| \beta_{2}\right)$ (e.g., message with $\operatorname{len}\left(m_{1} \| \beta_{2}\right)$ zero-bits). ${ }^{8}$ Alice encrypts this new message with the key $\beta_{2}$. Since Mallory has chosen the plaintext message, she has its length and hence obtains the keystream that is produced by $\beta_{1}$ (since the keystreams produced by $\beta_{2}$ and $\beta_{1}$ are identical). Using this, the $\operatorname{len}\left(m_{1}\right)$ and $E_{\beta_{1}}\left(m_{1} \| \beta_{2}\right)$, she recovers $\beta_{2}$. The attack requires $1 \mathrm{KP}$ (or 1 message length), $1 \mathrm{CP}$ (in which the 'plaintext' is actually 'extended plaintext'), the corresponding intercepted texts, and negligible time.

The attacker can continue recovering subsequent messages and keys. For example, after recovering $\beta_{2}$, given the message length $\operatorname{len}\left(m_{2}\right)$, the message $m_{2}$ and the key $\beta_{3}$ can be obtained. Subsequently, using $\beta_{3}$ and $\operatorname{len}\left(m_{3}\right)$, she can recover $m_{3}$ and $\beta_{4}$; and so on.

\subsection{Attacks in a Non-Related-Key Setting}

The stream cipher can also be attacked in a non-related-key setting. The attack works as follows.

Attack 3: In the example given in [2], while the CBS is formed from the ODNL, the leading zeroes of each number in the ODNL are discarded. When S works this way, the MSB of the keystream is 1 if the leading bits of the CBS are not discarded while forming the keystream (i.e., only the redundant trailing bits of the CBS are discarded). Given that the MSB of the keystream is 1, Mallory computes the MSB of the plaintext by complementing the MSB of the intercepted text. This attack recovers only one plaintext bit from the corresponding ciphertext bit, but requires practically zero time.

Even if Mallory does not know where the $\operatorname{len}\left(m_{i}\right)$ is inserted in the intercepted text (i.e., Assumption 2 is not made), she can mount Attack 3. If she knows len $\left(\operatorname{len}\left(m_{i}\right)\right)$ or len $\left(m_{i}\right)$, she can conclude that the recovered bit is a plaintext bit with probability $p \geq 1-1 /\left(d-\operatorname{len}\left(\operatorname{len}\left(m_{i}\right)\right)+1\right)$ (see Sect. 4$)$. This probability is high because of the following argument. The minimum length of $\beta_{i+1}$ is 4 bits because it has 4 subkeys. Since $d=\operatorname{len}\left(\operatorname{len}\left(m_{i}\right)\right)+\operatorname{len}\left(m_{i}\right)+\operatorname{len}\left(\beta_{i+1}\right)$, for a zero-length message, $d \geq 5$. Therefore, for any message, $d-\operatorname{len}\left(\operatorname{len}\left(m_{i}\right)\right)+1 \geq$ $5 \Rightarrow p \geq 0.8$. This attack, in addition to the requirements of Attack 3, requires message-dependent information in the form of $\operatorname{len}\left(\operatorname{len}\left(m_{i}\right)\right)$ or $\operatorname{len}\left(m_{i}\right)$. The success probability is at least 0.8 .

\footnotetext{
${ }^{8}$ In this paper, we use the placeholder name Mallory in place of the more common Eve because the attacker is not completely passive in Attack 2 (see also [15]).
} 
Attack 3 works regardless of whether the array $A[0, \ldots, N-1]$ is secret or not. The same is the case with the attack in Sect. 4 and Attacks 1 and 2. However, for Mallory to recover a message $m_{i}$ using a recovered key $\beta_{i}$, she needs to know $A[0, \ldots, N-1]$ to compute the keystream.

Attacks due to a poor choice of the array: Depending on how the array $A[0, \ldots, N-1]$ is chosen, we may have more attacks. For example, let us consider the following array containing elements from around the Feynman point in the decimal number sequence of $\pi$ :

$$
A[0, \ldots, 20]=\{9,9,6,0,5,1,8,7,0,7,2,1,1,3,4,9,9,9,9,9,9\} .
$$

Let $r=17$. The number of possibilities for the key is $21 \cdot C(21,17) \cdot 17 ! \cdot 2^{16} \approx$ $2^{81.3}$ - this is high enough to make a straightforward exhaustive key search nearly infeasible in practice. Suppose that the array is public. Since there are 6 even numbers in the array and $r=17$, the number of odd numbers in the ODNL is at least 11 . If there are exactly $t$ odd numbers $(1 \leq t \leq 11)$ in the ODNL, the probability that an odd number is the last digit in the ODNL is $t / 17$. Therefore, the probability that the last digit in the ODNL is odd is at least $11 / 17$. In other words, given that redundant trailing bits are not discarded during truncation of the CBS, the probability that the LSB of the extended ciphertext is complemented during transmission is at least $11 / 17$. One may, alternatively, exploit the presence of the unusually many 9's in the array in some clever manner. It may not be common for the users of the system to choose such number sequences, but a smaller bias may result when the array contains unequal numbers of even and odd digits (note that the array need not contain contiguous digits from the decimal representation of $\pi$ ).

\section{Conclusions and Open Problems}

In this paper, we presented several attacks on a cryptosystem patented by Artus [2]. The attacks are of extremely low data complexity and require nearly zero time. We also point out a major weakness in the key exchange scheme proposed in [2]. It is hoped that the results of this paper have shed light on how not to design ciphers and how the proposed cryptosystem has to be used in practice.

The patent also proposes a so-called "embodiment" of the cryptosystem in which Alice, instead of encrypting keys along with plaintexts, transmits the keys as pilot-messages. One can immediately see that this embodiment is also vulnerable to the attack of Sect. 4 and Attack 3 of Sect. 6 .

While it seems that some of the attacks presented in this paper could be easily averted (e.g., by using PKC for key exchange), it is also possible that there are better attacks in a non-related-key setting. As the flaws pointed out in this paper are serious enough, there was not enough motivation for us to work further in the non-related-key setting. However, if the cryptosystem is tweaked in future, so as to thwart the attacks in this paper, a more detailed analysis may be worthwhile carrying out. 


\section{References}

1. Anonymous, "Thank you Bob Anderson", Cypherpunks mailing list, 1994, available at http://web.archive.org/web/20080120083537/ http://cypherpunks . venona.com/date/1994/09/msg00304.html.

2. R.G.C. Artus, "METHOD AND SYSTEM FOR ENCRYPTION OF DATA", International Patent, publication number WO/2009/066313, 28 May 2009, available at http: //www . wipo . int/pctdb/en/wo . jsp?WO=2009066313.

3. E. Barkan, E. Biham, N. Keller, "Instant Ciphertext-Only Cryptanalysis of GSM Encrypted Communication", CRYPTO 2003 (D. Boneh, ed.), vol. 2729 of LNCS, pp. 600-616, Springer-Verlag, 2003.

4. W. Diffie, M.E. Hellman, "New Directions in Cryptography", IEEE Transactions on Information Theory, vol. 22, pp. 644-654, 1976.

5. O. Dunkelman, N. Keller, A. Shamir, "A Practical-Time Attack on the A5/3 Cryptosystem Used in Third Generation GSM Telephony", Cryptology ePrint Archive, Report 2010/013, 2010, available at http://eprint.iacr.org/2010/013.

6. I. Goldberg, D. Wagner, L. Green, "The (Real-Time) Cryptanalysis of A5/2", rump session of CRYPTO 1999, 1999

7. S. Indesteege, N. Keller, E. Biham, O. Dunkelman, B. Preneel, "A Practical Attack on KeeLoq", EUROCRYPT 2008 (N. Smart, ed.), vol. 4965 of LNCS, pp. 1-18, Springer-Verlag, 2008.

8. A. Kerckhoffs, "La cryptographie militaire", Journal des sciences militaires, vol. IX, pp. 5-83, January 1883, pp. 161-191, February 1883.

9. N. Koblitz, "Elliptic curve cryptosystems", Mathematics of Computation, vol. 48, pp. 203-209, 1987.

10. I. Mantin, "RC4", 2002, available at http://www.wisdom. weizmann.ac.il/ itsik/RC4/rc4.html.

11. Microchip Technology Inc., "KeeLoq Authentication Products", 2009, available at http://www.microchip.com/keeloq/.

12. V.S. Miller, "Use of Elliptic Curves in Cryptography", CRYPTO 1985 (H.C. Williams, ed.), vol. 218 of $L N C S$, pp. 417-426, Springer-Verlag, 1986.

13. R.L. Rivest, A. Shamir, L.M. Adleman, "A Method for Obtaining Digital Signatures and Public-Key Cryptosystems", Communications of the ACM, vol. 21(2), pp. 120-126, 1978.

14. R.L. Rivest, "Chaffing and Winnowing: Confidentiality without Encryption", April 1998, available at http://people.csail.mit.edu/rivest/Chaffing.txt.

15. B. Schneier, "Applied Cryptography", Second Edition, John Wiley \& Sons, January 1996.

16. C.E. Shannon, "Communication Theory of Secrecy Systems", Bell System Technical Journal, vol. 28-4, pp. 656-715, 1949.

17. Wikipedia, "KeeLoq", July 2010, available at http://en.wikipedia.org/wiki/KeeLoq. 